\title{
An $\alpha$ - Fixed Point Theorem in Complete Metric Spaces with Ordering by Iterations
}

\author{
T. Som ${ }^{1}$, A Choudhury ${ }^{1}$, B. S Choudhury ${ }^{2}$, Amaresh Kundu ${ }^{3, *}$ \\ ${ }^{1}$ Department of Applied Mathematics Indian Institute of Technology (BHU), Varanasi - 221005, India \\ ${ }^{2}$ Department of Mathematics, Bengal Engineering and Science University, Shibpur, Howrah - 711103, West Bengal, India \\ ${ }^{3}$ Department of Mathematics, Siliguri Institute of Technology, Darjeeling -734009, India \\ *Corresponding Author: kunduamaresh@yahoo.com
}

Copyright (C) 2014 Horizon Research Publishing All rights reserved.

\begin{abstract}
The aim of this present work is to generalize the notion of weakly isotone increasing mappings and prove an $\alpha$ fixed point theorems for $\alpha$-weakly isotone increasing self mappings which satisfy some inequality in complete metric spaces endow with partially ordering.
\end{abstract}

Keywords $\alpha$-Fixed Point, Ordered Metric Space, Complete Metric Spaces, $\alpha$-Weakly Isotone Increasing Mappings

\section{Introduction and Mathematical preliminaries}

The purpose of this paper is to prove an $\alpha$ - fixed point is an extensions of ordinary fixed points with the help of some function $\alpha$. It was introduced in [11] wherein a result on this was also established.

In partially ordered metric spaces the study of analytic fixed point theory is of relatively recent origin. Ran and Reurings [10] applied some results on partially ordered metric spaces to solving matrix equations. Over the time the theory has developed through important works like [4, 7-10].

Below are mathematical preliminaries required for the discussion in this paper.

Definition 1.1 Let $(X, \leqslant)$ be a partially ordered set. Then:

(a) elements $\mathrm{x}, \mathrm{y} \in \mathrm{X}$ are called comparable if $\mathrm{x} \leqslant \mathrm{y}$ or $\mathrm{y} \leqslant \mathrm{x}$ holds;

(b) a subset $\mathrm{K}$ of $\mathrm{X}$ is said to be well ordered if every two elements of $\mathrm{K}$ are comparable;

(c) a mapping $\mathrm{T}: \mathrm{X} \rightarrow \mathrm{X}$ is called nondecreasing w.r.t. $\leqslant$ if $\mathrm{x} \leqslant \mathrm{y}$ implies $\mathrm{Tx} \leqslant \mathrm{Ty}$.

Definition1.2 Let $X$ be a nonempty set. Then $(X, d, \preccurlyeq)$ is called a partially ordered metric space if:

i. $\quad(X, d)$ is a metric space, and (ii) $(X, \leqslant)$ is a partially ordered set.

Definition 1.3 Let $(X, d, \leqslant)$ be a partially ordered metric space. Then a sequence $\left\{x_{n}\right\}$ in $X$ is said to be non-decreasing sequence in $\mathrm{X}$ if $\mathrm{x}_{1} \leqslant \mathrm{x}_{2} \leqslant \ldots \leqslant \mathrm{x}_{\mathrm{n}}$ for all $\mathrm{n} \in \mathrm{N}$.

Definition 1.4 [9] Let $(X, d, \leqslant)$ be a partially ordered metric space. We say that $X$ is regular if the following hypothesis holds: if $\left\{z_{\mathrm{n}}\right\}$ is a non-decreasing sequence in $\mathrm{X}$ with respect to $\leqslant$ such that $\mathrm{z}_{\mathrm{n}} \rightarrow \mathrm{z} \in \mathrm{X}$ as $\mathrm{n} \rightarrow \infty$,

then $\mathrm{z}_{\mathrm{n}} \leqslant \mathrm{z}$ for all $\mathrm{n} \in \mathrm{N}$.

Definition 1.5 ( $\alpha$-fixed point) Let $\alpha: X \rightarrow X$, a point $\mathrm{X}$ is said to be a $\alpha$-fixed point of a mapping $\mathrm{T}: \mathrm{X} \rightarrow \mathrm{X}$ if $\alpha \mathrm{ox}$ $=\alpha \mathrm{oT}(\mathrm{x})$.

Definition 1.6 [9] Let $(X, \leqslant)$ be a partially ordered set. A pair of mappings $S, T: X \rightarrow X$ is said to be weakly increasing if $S x$ $\leqslant \mathrm{T}$ Sx and $\mathrm{T} x \leqslant$ ST $\mathrm{x}$ for all $\mathrm{x} \in \mathrm{X}$.

Definition 1.7 [9] Let $(X, \leqslant)$ be a partially ordered set and let $S, T: X \rightarrow X$ be two mappings. The mapping $S$ is said to be $T$ -weakly isotone increasing if for all $\mathrm{x} \in \mathrm{X}$ we have $\mathrm{Sx} \leqslant \mathrm{TSx} \leqslant \mathrm{STSx}$.

Definition 1.8 ( $\alpha$-weakly isotone increasing) Let $(X, \leqslant)$ be a partially ordered set and let $\alpha: X \rightarrow X$, and $S$, T be two self mappings of $X$. The mapping $S$ is said to be $T, \alpha$-weakly isotone increasing if for all $\mathrm{x} \in \mathrm{X}$ we have $(\alpha \circ \mathrm{S}) \mathrm{x} \leqslant(\alpha \circ \mathrm{T})(\alpha \circ \mathrm{S}) \mathrm{x}$ $\leqslant(\alpha \circ S)(\alpha \circ \mathrm{o})(\alpha \circ S) x$.

Remark1.2 If $\mathrm{S}, \mathrm{T}: \mathrm{X} \rightarrow \mathrm{X}$ are weakly increasing, then $\mathrm{S}$ is $\mathrm{T}$-weakly isotone increasing.

In the forth coming section we prove a common $\alpha$-fixed points result in metric spaces. 


\section{Main Results}

The common $\alpha$-fixed point result in partially ordered metric spaces satisfying the isotone increasing property, goes as follows.

Theorem 2.1 Let $(X, \leq)$ be a partially ordered set and suppose that there exists a metric $d$ on $X$ such that $(X, d)$ is a complete metric space. Let $\alpha: X \rightarrow X$ and let $\mathrm{T}$ and $\mathrm{S}$ be two self mappings of $(\mathrm{X}, \mathrm{d})$ such that for comparable $\mathrm{X}, \mathrm{y} \in \mathrm{X}$.

$$
\begin{gathered}
\operatorname{ad}(\alpha \mathrm{oT}(\mathrm{x}), \alpha \mathrm{oS}(\mathrm{y}))+\operatorname{bd}(\alpha \mathrm{oT}(\mathrm{x}), \alpha(\mathrm{x}))+\mathrm{cd}(\alpha(\mathrm{y}), \alpha \mathrm{oS}(\mathrm{y}))-\min \{\mathrm{d}(\alpha \mathrm{oT}(\mathrm{x}), \alpha(\mathrm{y})), \mathrm{d}(\alpha \mathrm{oS}(\mathrm{y}), \alpha(\mathrm{x}))\} \\
\leq \mathrm{q} \max \left\{\mathrm{d}(\alpha(\mathrm{x}), \alpha(\mathrm{y})), \mathrm{d}(\alpha \mathrm{oT}(\mathrm{x}), \alpha(\mathrm{x})), \mathrm{d}(\alpha(\mathrm{y}), \alpha \mathrm{oS}(\mathrm{y})), \frac{1}{2} \mathrm{~d}(\alpha \mathrm{oT}(\mathrm{x}), \alpha(\mathrm{y}))\right\}
\end{gathered}
$$

where $a, b, c \geq 0, q>0$ with $a>q$.

We suppose the following hypotheses:

(a) $\mathrm{S}$ is T, $\alpha$-weakly isotone increasing,

(b) $\mathrm{X}$ is regular.

Then $\mathrm{S}$ and $\mathrm{T}$ have a unique common $\alpha$-fixed point.

Proof : Let $\mathrm{x}_{\mathrm{o}} \in \mathrm{X}$ be an arbitrary point. We generate a sequence $\left\{\mathrm{x}_{\mathrm{n}}\right\}$ with respect to $\alpha$ such that

$$
\mathrm{x}_{2 \mathrm{n}+2}=\alpha \mathrm{oT}\left(\mathrm{x}_{2 \mathrm{n}+1}\right)=\mathrm{T}_{\alpha}\left(\mathrm{x}_{2 \mathrm{n}+1}\right) \text { and } \mathrm{x}_{2 \mathrm{n}+1}=\alpha \mathrm{oS}\left(\mathrm{x}_{2 \mathrm{n}}\right)=\mathrm{S}_{\alpha}\left(\mathrm{x}_{2 \mathrm{n}}\right) \text { for } \mathrm{n}=0,1,2, \ldots .
$$

Let $\mathrm{d}_{\mathrm{n}}=\mathrm{d}\left(\alpha \mathrm{o}\left(\mathrm{x}_{\mathrm{n}}\right), \alpha \mathrm{o}\left(\mathrm{x}_{\mathrm{n}+1}\right)\right)>0, \mathrm{n}=0,1,2, \ldots$

Since $\mathrm{S}_{\alpha}$ is $\mathrm{T}_{\alpha}$-weakly isotone increasing, we have

$$
\begin{gathered}
x_{1} \leq \alpha \mathrm{oS}\left(\mathrm{x}_{0}\right) \leq \alpha \mathrm{oT}\left(\alpha \mathrm{oS}\left(\mathrm{x}_{0}\right)\right)=\alpha \mathrm{oT}\left(\mathrm{x}_{1}\right)=x_{2} \leq(\alpha \mathrm{oS})\left(\alpha \mathrm{oT}\left(\alpha \mathrm{oS}\left(\mathrm{x}_{0}\right)\right)\right)=\alpha \operatorname{oS}\left(\alpha \mathrm{oT}\left(\mathrm{x}_{1}\right)\right)=\alpha \mathrm{oS}\left(\mathrm{x}_{2}\right)=x_{3} \\
x_{3} \leq \alpha \mathrm{oS}\left(\mathrm{x}_{2}\right) \leq \alpha \mathrm{oT}\left(\alpha \mathrm{oS}\left(\mathrm{x}_{2}\right)\right)=\alpha \mathrm{oT}\left(\mathrm{x}_{3}\right)=x_{4} \leq(\alpha \mathrm{oS})\left(\alpha \mathrm{oT}\left(\alpha \mathrm{oS}\left(\mathrm{x}_{2}\right)\right)\right)=\alpha \mathrm{oS}\left(\alpha \mathrm{oT}\left(\mathrm{x}_{3}\right)\right)=\alpha \mathrm{oS}\left(\mathrm{x}_{4}\right)=x_{5} \ldots \ldots .
\end{gathered}
$$

and continuing this process we get, $x_{1} \leq x_{2} \leq x_{3} \ldots \ldots \ldots \leq x_{n} \leq x_{n+1} \leq \ldots \ldots \ldots$

Since $x_{2 n} \leq x_{2 n+1}$ for all $\mathrm{n}=0,1,2 \ldots$, from (ii), putting $x=x_{2 n+1}$ and $y=x_{2 n}$, we have

$$
\begin{gathered}
{\left[\operatorname{ad}\left(\alpha o T\left(x_{2 n+1}\right), \alpha o S\left(x_{2 n}\right)\right)+\operatorname{bd}\left(\alpha o T\left(x_{2 n+1}\right), x_{2 n+2}\right)+\operatorname{cd}\left(x_{2 n}, \alpha o S\left(x_{2 n}\right)\right)\right.} \\
\left.-\min \left\{d\left(\alpha o T\left(x_{2 n+1}\right), x_{2 n}\right), d\left(\alpha o S\left(x_{2 n}\right), x_{2 n+1}\right)\right\}\right] \\
\leq \operatorname{qmax}\left\{d\left(x_{2 n+1}, x_{2 n}\right), d\left(\alpha o T\left(x_{2 n+1}\right), x_{2 n+1}\right)\right. \\
\left.\quad d\left(x_{2 n}, \alpha o S\left(x_{2 n}\right)\right), \frac{1}{2} d\left(\alpha o T\left(x_{2 n+1}\right), x_{2 n}\right)\right\}
\end{gathered}
$$

or,

$$
\begin{gathered}
{\left[\operatorname{ad}\left(x_{2 n+2}, x_{2 n+1}\right)+b d\left(x_{2 n+2}, x_{2 n+1}\right)+\operatorname{cd}\left(x_{2 n}, x_{2 n+1}\right)-\min \left\{d\left(x_{2 n}, x_{2 n+2}\right), d\left(x_{2 n+1}, x_{2 n+1}\right)\right\}\right]} \\
\leq q \max \left\{d\left(x_{2 n+1}, x_{2 n}\right), d\left(x_{2 n+2}, x_{2 n+1}\right), d\left(x_{2 n}, x_{2 n+1}\right), \frac{1}{2} d\left(x_{2 n+2}, x_{2 n}\right)\right\}
\end{gathered}
$$

or,

$$
\operatorname{ad}_{2 \mathrm{n}+1}+\mathrm{bd}_{2 \mathrm{n}+1}+\mathrm{cd}_{2 \mathrm{n}}-\min \left\{\left(\mathrm{d}_{2 \mathrm{n}}+\mathrm{d}_{2 \mathrm{n}+1}\right), 0\right\} \leq \mathrm{q} \max \left\{\mathrm{d}_{2 \mathrm{n}}, \mathrm{d}_{2 \mathrm{n}+1}, \frac{1}{2}\left(\mathrm{~d}_{2 \mathrm{n}}+\mathrm{d}_{2 \mathrm{n}+1}\right)\right\}
$$

Putting $\mathrm{k}=\max \left\{\mathrm{d}_{2 \mathrm{n}}, \mathrm{d}_{2 \mathrm{n}+1}\right\}$, we have $\mathrm{d}_{2 \mathrm{n}} \leq \mathrm{k}$ and $\mathrm{d}_{2 \mathrm{n}+1} \leq \mathrm{k}$ implying

$$
\frac{1}{2}\left(\mathrm{~d}_{2 \mathrm{n}}+\mathrm{d}_{2 \mathrm{n}+1}\right) \leq \mathrm{k} \text {. }
$$

Therefore,

$$
\max \left\{\mathrm{d}_{2 \mathrm{n}}, \mathrm{d}_{2 \mathrm{n}+1}, 1 / 2\left(\mathrm{~d}_{2 \mathrm{n}}+\mathrm{d}_{2 \mathrm{n}+1}\right)\right\} \leq \mathrm{k}=\max \left\{\mathrm{d}_{2 \mathrm{n}}, \mathrm{d}_{2 \mathrm{n}+1}\right\}
$$

Thus we have from (iii),

$$
\text { (a+b) } d_{2 n+1}+\operatorname{cd}_{2 n} \leq q \max \left\{d_{2 n}, d_{2 n+1}\right\}
$$

If $\mathrm{d}_{2 \mathrm{n}} \leq \mathrm{d}_{2 \mathrm{n}+1}$ then $(\mathrm{a}+\mathrm{b}) \mathrm{d}_{2 \mathrm{n}+1}+\mathrm{cd}_{2 \mathrm{n}} \leq \mathrm{qd}_{2 \mathrm{n}+1}$

$$
\Rightarrow(a+b-q) d_{2 n+1} \leq-c d_{2 n}
$$




$$
\Rightarrow \mathrm{d}_{2 \mathrm{n}+1} \leq \mathrm{pd}_{2 \mathrm{n}} \text { where } \mathrm{p}=\frac{-c}{a+b-q}<1
$$

If $d_{2 n+1} \leq d_{2 n}$, then

$$
\begin{gathered}
(\mathrm{a}+\mathrm{b}) \mathrm{d}_{2 \mathrm{n}+1}+\mathrm{cd}_{2 \mathrm{n}} \leq \mathrm{qd}_{2 \mathrm{n}} \\
\Rightarrow \mathrm{d}_{2 \mathrm{n}+1} \leq \frac{q-c}{a+b} \mathrm{~d}_{2 \mathrm{n}} \\
\Rightarrow \mathrm{d}_{2 \mathrm{n}+1} \leq \mathrm{pd}_{2 \mathrm{n}} \text { where } \mathrm{p}=\frac{q-c}{a+b}<1
\end{gathered}
$$

Therefore,

$$
\mathrm{d}_{2 \mathrm{n}+1} \leq \mathrm{pd} \mathrm{d}_{2 \mathrm{n}} \leq \mathrm{p}^{2} \mathrm{~d}_{2 \mathrm{n}-1} \leq \ldots \ldots \ldots \ldots \ldots \ldots \mathrm{p}^{2 \mathrm{n}+1} \mathrm{~d}_{\mathrm{o}} \rightarrow 0 \text { as } \mathrm{n} \rightarrow \infty .
$$

Thus $\left\{\mathrm{x}_{2 \mathrm{n}}\right\}$ is a Cauchy sequence in $\mathrm{X}$ and since $\mathrm{X}$ is complete, there exists a point $\mathrm{u} \in \mathrm{X}$ such that $\left\{\mathrm{x}_{2 \mathrm{n}}\right\}$ converges to $u$. Hence

$$
\lim (\alpha \mathrm{oT})\left(\mathrm{x}_{2 \mathrm{n}+1}\right)=\lim \mathrm{x}_{2 \mathrm{n}+2}=\mathrm{u}
$$

and

$$
\lim (\alpha \mathrm{oS})\left(\mathrm{x}_{2 \mathrm{n}}\right)=\lim \mathrm{x}_{2 \mathrm{n}+1}=\mathrm{u} .
$$

Since $\left\{\mathrm{x}_{2 \mathrm{n}}\right\}$ is a nondecreasing sequence, if $\mathrm{X}$ is regular, it follows that $x_{2 n} \leq u$ for all $\mathrm{n}$.

Now putting $x=x_{2 n+1}$ and $y=u$ in (ii), we have

$$
\begin{gathered}
{\left[\operatorname{ad}\left((\alpha \circ \mathrm{O}) \mathrm{x}_{2 \mathrm{n}+1},(\alpha \mathrm{oS}) \mathrm{u}\right)+\operatorname{bd}\left(\mathrm{x}_{2 \mathrm{n}+1},(\alpha \mathrm{oT}) \mathrm{x}_{2 \mathrm{n}+1}\right)+\operatorname{cd}(\mathrm{u},(\alpha \mathrm{oS}) \mathrm{u})-\right.} \\
\left.\min \left\{\mathrm{d}\left((\alpha \mathrm{oT})\left(\mathrm{x}_{2 \mathrm{n}+1}\right), \mathrm{u}\right), \mathrm{d}\left((\alpha \mathrm{oS}) \mathrm{u}, \mathrm{x}_{2 \mathrm{n}+1}\right)\right\}\right] \\
\leq \mathrm{q} \max \left\{\mathrm{d}\left(\mathrm{x}_{2 \mathrm{n}+1}, \mathrm{u}\right), \mathrm{d}\left((\alpha \mathrm{O} \mathrm{T})\left(\mathrm{x}_{2 \mathrm{n}+1}\right), \mathrm{x}_{2 \mathrm{n}+1}\right), \mathrm{d}(\mathrm{u},(\alpha \mathrm{oS}) \mathrm{u}), \frac{1}{2} \mathrm{~d}\left((\alpha \mathrm{oT})\left(\mathrm{x}_{2 \mathrm{n}+1}\right), \mathrm{u}\right)\right\}
\end{gathered}
$$

In the limiting case, we have

$$
\begin{gathered}
\operatorname{ad}\left(u, \alpha_{0} S(u)\right)+b d(u, u)+c d(u, \alpha o S(u))-\min \{d(u, u), d(u, \alpha o S(u))\} \\
\leq \mathrm{q} \max \left\{d(u, u), d(u, u), d(u, \alpha o S(u)), \frac{1}{2} d(u, u)\right\}
\end{gathered}
$$

or, $(a+c-q) d(u, \alpha o S(u)) \leq 0$

or, $\alpha \mathrm{oS}(\mathrm{u})=\mathrm{u}$.

Since $\mathrm{a}>1+\mathrm{q}$. Thus $\mathrm{u}$ is a fixed point of $\mathrm{S}$.

Similarly by considering $\mathrm{x}=\mathrm{u}, \mathrm{y}=\mathrm{x}_{2 \mathrm{n}}$ we can get $\alpha o u=\alpha o T(\mathrm{u})$. Thus $\mathrm{u}$ is a common $\alpha$-fixed point of $\mathrm{T}$ and $\mathrm{S}$.

From (ii) it is easy to show the uniqueness of the common $\alpha$-fixed point and this completes the proof.

\section{REFERENCES}

[1] Meir,A. and Keeler,E. , A theorem on contraction mappings, J.Math.Anal.Appl. 28(1969), 326-329.

[2] Sessa,S., On a weak commutativity condition in fixed point considerations, Publ.Inst. Math. 32(46) (1982), 149-153.

[3] Som,T., A few common fixed point results for compatible mappings, Bull.Cal.Math. Soc.95 (2003), 307-312.

[4] Abbas, M., Nazir, T. and Radenovic, S., Common fixed points of four maps in partially ordered metric spaces, Appl. Math. Lett. (2011), doi:10.1016/j.aml.2011.03.038.

[5] Babu, G.V.R. and Vara Prasad, K.N.V.V., Common fixed point theorems of different compatible type mappings using Ciric's contraction type condition, Math. Commun., 11 (2006) 87-102. 
[6] Banach, S., Sur les op'erations dans les ensembles abstraits et leur applications aux 'equations int'egrales, Fund Math., 3 (1922), 133-181.

[7] Choudhury, B. S. and Kundu, A., A coupled coincidence point result in partially ordered metric spaces for compatible mappings, Nonlinear Analysis, TMA 73, (2010) 2524-253.

[8] Harjani, J. and Sadarangani, K., Fixed point theorems for weakly contractive mappings in partially ordered sets, Nonlinear Anal. TMA, 71 (2009) 3403-3410.

[9] Nashine, H. K., Kadelburg, Z. and Radenovic, S., Common fixed point theorems for weakly isotone increasing mappings in ordered partial metric spaces, Math. Comput. Modelling, in press, doi:10.1016/j.mcm.2011.12.019.

[10] Ran, A.C.M. and Reurings, M.C.B., A fixed point theorem in partially ordered sets and some applications to matrix equations, Proc. Amer. Math. Soc., 132(5) (2004),1435- 1443.

[11] Srivastava A and Pawar A, $\alpha$-compatible mappings and $\alpha$-compatible mappings of type (A) , Jr. Indian Acad. Math., 26 (2) 2006 , 409-425. 